\title{
A CHARACTERIZATION OF THE SPHERE IN TERMS OF SINGLE-LAYER POTENTIALS
}

\author{
HENRIK SHAHGHOLIAN
}

(Communicated by J. Marshall Ash)

\begin{abstract}
Let $\Omega$ be a bounded smooth domain in $\mathbb{R}^{n}$, and suppose the singlelayer potential of $\partial \Omega$ coincides for $y \notin \bar{\Omega}$ with the function $c|y|^{-1}(c>0)$. Then $\partial \Omega$ is a sphere centered at the origin.
\end{abstract}

Throughout this paper we assume $\Omega \subset \mathbb{R}^{3}$ (the case $\mathbb{R}^{n}$ is similar) is a bounded domain, and the boundary $\partial \Omega$ is smooth enough that for

$$
u(y)=\int_{\partial \Omega} \frac{d \sigma_{x}}{|x-y|} \quad \forall y \in \mathbb{R}^{n},
$$

one has

$$
4 \pi=\frac{\partial u}{\partial n^{-}}-\frac{\partial u}{\partial n^{+}} \quad \text { on } \partial \Omega,
$$

where $d \sigma_{x}$ denotes the surface measure, + indicates the limit from the exterior and - the limit from the interior (see [K, p. 164]), and $n$ is the outward normal vector to $\partial \Omega$, which we assume to exist. If $\Omega$ has this property, then we say $\Omega$ is smooth.

Theorem. Let $\Omega$ be a bounded smooth domain in $\mathbb{R}^{3}$, and suppose for some $c>0$

$$
\int_{\partial \Omega} \frac{d \sigma_{x}}{|x-y|}=\frac{c}{|y|} \quad \forall y \neq \bar{\Omega} .
$$

Then, $\partial \Omega$ is a sphere centered at the origin.

Proof. Since the single-layer potential is continuous in $\mathbb{R}^{3}$ (see $[K$, p. 160]) (3) implies $0 \in \Omega$. Therefore we can take two balls $B_{1}, B_{2}$ both centered at the origin, such that $B_{1}$ is the largest ball in $\Omega$ and $B_{2}$ is the smallest ball containing $\Omega$. Now the idea is to show that $\partial B_{1}=\partial B_{2}=\partial \Omega$. Now let $y^{1} \in \partial B_{1} \cap \partial \Omega$ and $y^{2} \in \partial B_{2} \cap \partial \Omega$, then $\left|y^{2}\right| \geq\left|y^{1}\right|$. Thus it suffices to prove $\left|y^{2}\right| \leq\left|y^{1}\right|$. Define $u$ to be the function represented by (1). Then by the maximum principle (since $u$ is harmonic in $\Omega$ ) $\max u$ in $\Omega$ is attained on

Received by the editors January 7, 1991.

1980 Mathematics Subject Classification (1985 Revision). Primary 31 B20.

Key words and phrases. Single-layer potential, harmonic function, mean value property.

(C) 1992 American Mathematical Society $0002-9939 / 92 \$ 1.00+\$ .25$ per page 
$\partial \Omega$, hence by (3) at $y^{1}$. Similarly, $\min u$ in $\Omega$ is attained at $y^{2}$; therefore,

$$
\frac{\partial u}{\partial n^{-}}\left(y^{1}\right) \geq 0 \text { and } \frac{\partial u}{\partial n^{-}}\left(y^{2}\right) \leq 0 .
$$

Moreover, at $y^{1}$ and $y^{2}$ we have

$$
\frac{\partial}{\partial n^{+}}=\frac{\partial}{\partial|y|},
$$

which applied to (2) yields

$$
4 \pi=\frac{\partial u}{\partial n^{-}}-\frac{\partial u}{\partial n^{+}}=\frac{\partial u}{\partial n^{-}}-c \frac{\partial|y|^{-1}}{\partial|y|} \geq \frac{c}{|y|^{2}} \text { at } y^{1},
$$

i.e., $\left|y^{1}\right| \geq \sqrt{c / 4 \pi}$. Similarly

$$
4 \pi=\frac{\partial u}{\partial n^{-}}-\frac{\partial u}{\partial n^{+}}=\frac{\partial u}{\partial n^{-}}-c \frac{\partial|y|^{-1}}{\partial|y|} \leq \frac{c}{|y|^{2}} \quad \text { at } y^{2},
$$

i.e., $\left|y^{2}\right| \leq \sqrt{c / 4 \pi}$. This completes the proof.

Remark. As far as the proof of the theorem goes, we do not need equation (2) to hold on the entire boundary of $\Omega$, but just at the points on $\partial \Omega$ where $u$ attains its maximum and minimum.

The theorem can very easily be generalized to the case in which $\Omega$ is a union of disjoint domains or in which the density is assumed to be radially increasing. These generalizations, in the context of the volume potential, are dealt with in [ASZ].

We state these as corollaries, omitting the simple proofs.

Corollary 1. Let

$$
\int_{\Omega} \frac{d \sigma_{x}}{|x-y|}=\sum_{j=1}^{m} \frac{c_{j}}{\left|x^{j}-y\right|} \quad \forall y \notin \bar{\Omega},
$$

where $\Omega=\bigcup_{1}^{m} \Omega_{j},\left\{\Omega_{j}\right\}$ are pairwise disjoint smooth domains, and $x^{j} \in \Omega_{j}$. Then, $\partial \Omega_{j}$ is a sphere centered at $x^{j}, \forall_{j}$.

Corollary 2. Let $f=f(|x|)$ be a continuous increasing function defined on $\mathbb{R}^{3}$, and suppose

$$
\int_{\partial \Omega} \frac{f(|x|) d \sigma_{x}}{|x-y|}=\frac{c}{|y|} \quad \forall y \notin \bar{\Omega} .
$$

Then $\partial \Omega$ is a sphere centered at the origin.

\section{REFERENCES}

[ASZ] D. Aharonov, M. M. Schiffer, and L. Zalcman, Potato Kugel, Israel J. Math. 40 (1981), 331-339.

[K] O. D. Kellogg, Foundations of potential theory, 4th printing, Ungar, New York, 1970.

Department of Mathematics, Royal Institute of Technology, S-100 44 Stockholm 70, SWEDEN 